\title{
Perspectives
}

\section{Human brain evolution, theories of innovation, and lessons from the history of technology}

\author{
ALFRED GIERER \\ Max-Planck-Institute for Developmental Biology, Spemannstrasse 35, D-72076 Tübingen, Germany \\ (Fax,49-7071-601448; Email, alfred.gierer@tuebingen.mpg.de)
}

Biological evolution and technological innovation, while differing in many respects, also share common features. In particular, the implementation of a new technology in the market is analogous to the spreading of a new genetic trait in a population. Technological innovation may occur either through the accumulation of quantitative changes, as in the development of the ocean clipper, or it may be initiated by a new combination of features or subsystems, as in the case of steamships. Other examples of the latter type are electric networks that combine the generation, distribution, and use of electricity, and containerized transportation that combines standardized containers, logistics, and ships. Biological evolution proceeds, phenotypically, in many small steps, but at the genetic level novel features may arise not only through the accumulation of many small, common mutational changes, but also when distinct, relatively rare genetic changes are followed by many further mutations. New evolutionary directions may be initiated by, in particular, some rare combinations of regulatory sections within the genome.

The combinatorial type of mechanism may not be a logical prerequisite for biological innovation, but it can be efficient, especially when novel features arise out of already highly developed systems. Such is the case with the evolution of general, widely applicable capabilities of the human brain. Hypothetical examples include the evolution of strategic thought, which encompasses multiple self-representations, cognition-based empathy, meta-levels of abstraction, and symbolic language. These capabilities of biologically modern man may have been initiated, perhaps some 150,000 years ago, by one or few accidental but distinct combinations of modules and subroutines of gene regulation which are involved in the generation of the neural network in the cerebral cortex. This hypothesis concurs with current insights into the molecular biology of the combinatorial and hierarchical facets of gene regulation that underlie brain development. A theory of innovation encompassing technological as well as biological development cannot per se dictate alternative explanations of biological evolution, but it may help in adding weight and directing attention to notions outside the mainstream, such as the hypothesis that few distinct genetic changes were crucial for the evolution of modern man.

\section{Phenotypic gradualism versus genotypic distinctness}

When Darwin (1859) proposed his evolutionary theory of the origin of species based on variation and selection according to what was later termed "fitness", he insisted that evolution was a gradual process involving many small steps: "As natural selection acts solely by accumulating slight, successive, favourable variations ... it can act only by very short and slow steps. ... If it could be demonstrated that any complex organism existed which could not possibly have been formed by numerous successive slight modifications my theory would absolutely break down". Such comments display Darwin's bias in favour of gradualism pure, possibly motivated by the urge to disprove fundamentalist theories of biblical creationism (Mayr 1988). And yet, in the Middle Ages, the more enlightened branches of theology were already insisting on the need to interpret the story of creation in abstract terms. Thierry of Chartres proposed as early as the 12th century that God had created the elements and the laws they follow, and that everything, including life, then developed by itself according to the laws of nature (Haring 1955). Darwin was hardly more radical in his evolutionary views than was Thierry seven hundred years earlier. Nor does the assumption of distinct steps in evolution necessarily support creationism. The probability of distinct developments, though very low per individual, need not be too low to occur in large populations over many generations without the aid of miracles.

At present, overly gradualistic notions remain popular in parts of the scientific community, but in fact a discus- 
sion of evolutionary mechanisms in terms of modern systems theory and molecular genetics does not map one-toone onto the traditional alternatives of gradual versus discontinuous processes. Cells and organisms are biochemical systems following the rules of non-linear molecular interactions. These, in turn, allow for sudden or very fast changes resulting from the accumulation of rapidly succeeding small steps with self-enhancing features. Furthermore, mechanisms of bifurcation and de-novo pattern formation may lead, for instance, to strikingly different developments in parts of an initially near-uniform area. Such self-organizing processes can be initiated by the gradual accumulation of certain molecules, or a gradual increase in tissue size, when passing a distinct threshold. There is, therefore, no logical need to generally infer big steps as causes for big changes. However, in light of molecular genetics, we must also consider mechanisms which may appear gradual phenotypically but which are distinct with respect to the genetic events that initiate them, as well as the corresponding explanatory logic.

\section{Molecular genetics of evolutionary change}

There is no doubt about the significance of small quantitative changes in the evolution of higher organisms. They may result, for instance, from the accumulation of point mutations (though even a single point mutation can lead to major changes, such as sickle cell anemia resulting from a single amino acid replacement in hemoglobin). However, an essential role in evolution is also played by major genetic alterations such as duplications, combinations, and transpositions of DNA segments within the genome (see Alberts et al 2002). The segments involved may contain not only genes, parts of genes and groups of genes coding for proteins, but also regulatory sections of the genome affecting gene expression, i.e. the synthesis of specific proteins. Such changes are not inconsistent with phenotypic gradualism, but in terms of molecular genetics they could hardly be categorized as "gradual".

In this context, the regulatory nucleotide sequences in the DNA deserve particular attention. Their function depends in a subtle manner on their combination and on their position within the genome. They bind regulatory proteins; acting as "transcription factors", the combinations of bound proteins regulate the activation of (mostly nearby, but under certain conditions also fairly distant) protein-coding genes (see Alberts et al 2002). Many of them are flanked by whole batteries of activating and inhibiting "cis-regulatory sequences": "Therein lies the experimental path to understanding the organization of the genomic program for development" (Arnone and Davidson 1997).

The function of such sets of regulatory sequences has been compared, with good reason, to electronic micro- processing: they process the information contained in the set of regulatory proteins that characterizes the type and position of cells, as well as the stage and other developmental features of the organism, into the corresponding pattern of gene expression (figure 1). This leads to further development and eventually to the maintenance of the adult system.

The network of gene regulation is certainly complex, but not without internal order. Combinatorial and hierarchical features of developmental regulation postulated some thirty years ago (see Gierer 1973) have been demonstrated and characterized by a large body of evidence. Combined into sets, regulatory sequences with activating and inhibiting functions exhibit modular as well as synergetic characteristics. A protein-coding gene can be activated in different developmental contexts, serving different functions in different subsystems. Any given phenotype is the result of the cooperation of many genes. And there are hierarchical characteristics, with certain genes controlling gross features, such as the body plan.

These mechanisms are only part of a wider repertoire of developmental regulations including "post-transcriptional" control beyond gene activation, and the operation of signalling pathways via intercellular space. Various components of gene expression interact in many ways, and it is often the resultant products that then feed back into gene regulation. The network of gene expressions and gene products involved in the basic processes of embryogenesis is of almost discouraging complexity. One example is the pagefilling chart shown in a paper by twenty-five authors for the regulation of endomesodermal specification (Davidson et al 2002). And yet, the network of "microprocessing" gene regulation as illustrated in the highly simplified sketch of figure 1 is important and prototypical for developmental regulation at large: the feedback of products of gene regulation into the regulating genes allows for multiple stable states of cells with one and the same genome, and this, in turn, is the prerequisite of spatial-temporal cell differentiation in the course of the development of a multicellular organism.

How are the regulatory genomic features related to evolutionary processes? This is an emerging field of research with many more questions than answers. One of the basic processes is the recruitment of existing regulatory pathways into a newly evolving context (Wilkins 2002; Pires daSilva and Sommer 2003). In the model case of the evolution of eyespots in the butterfly wing, it seems that the "co-option" of a whole regulatory network is involved (Keys et al 1999). The molecular mechanisms remain disputed, but genetic changes affecting the regulatory sequences (especially the enhancers) feature prominently in discussion. Generally, there is increasing evidence for the evolutionary role of regulatory sequences, in addition to mutations in protein-coding genes (see Tautz 2000). 
Simple regulatory sites may evolve by point mutations alone, and sets of them could evolve consecutively, but the formation of complex arrays of protein-coding as well as of regulatory sequences may also involve duplications and transpositions of DNA-sections that give rise to new combinations within the same genome (see Alberts et al 2002). Duplicated protein-coding genes may follow different directions during further changes and acquire new functions, often involving cooperation with other sets of genes. Regulatory sections may be shifted to other positions in the genome; such an introduction into a new con- text can result in a novel combination of regulatory units, with modular as well as synergetic effects on specifying the proteins to be synthesized, which may entail many indirect consequences for cells and organisms. Conceivably, such accidental but distinct genetic events could, on rare occasions, initiate a new direction for evolution; the phenotypic effects may be small at the outset but the single initiating step may then be followed by many more common mutations such as nucleotide substitutions, deletions, or additions, giving gradual rise to striking effects in the long run.

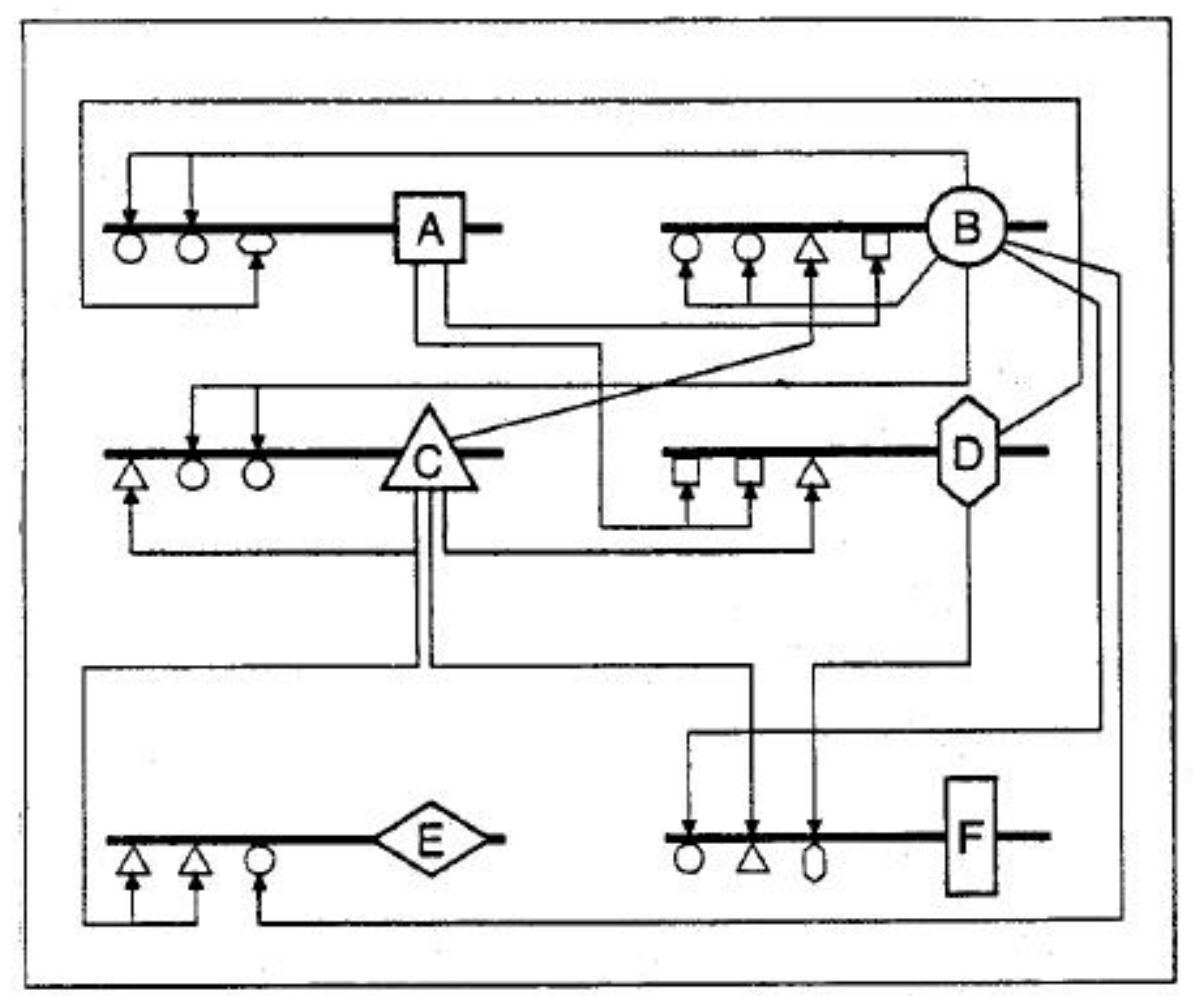

Figure 1. Modular organization of gene regulation. The genome contains, aside from genes coding for proteins (upper case letters A, B, etc in squares, triangles, etc), regulatory areas with binding sites (symbolized by small squares, triangles, etc) which bind regulatory proteins A-D. The set of bound sites exerts activating as well as inhibiting effects on the expression of (mostly nearby) genes. The synthesis of regulatory proteins involved in this regulation is itself regulated by the direct $(\mathrm{C})$ and indirect feedback of the regulatory proteins on their own synthesis $(\mathrm{A}-\mathrm{D})$. This allows for different stable states of cell differentiation, characterized by specific combinations of regulatory proteins. These, in turn, activate and inhibit the expression of many other protein-coding genes ( $\mathrm{E}, \mathrm{F}$, etc). The areas combining regulatory DNA-sequences in a modular manner may be considered as microprocessors of information on celltype, state, and position of development as contained in the specific combination of regulatory proteins, leading to the developmental regulation of gene expression. This scheme cannot encompass the spectrum of mechanisms (reviewed by Pires daSilva and Sommer 2003) that includes, for instance, cofactor binding to regulatory molecules rather than to DNA directly, and it addresses only one of the aspects of developmental regulation at large. Nevertheless, it is prototypical for its logical core which emphasizes the combinatorial characteristics and self-regulatory features of gene regulation in the course of development. 


\section{Possible accidental yet singular genetic change in human brain evolution}

Let us now consider the chain of processes by which genes control the development of the brain and thereby give rise to its functional capabilities. The neo-cortex of man contains some hundreds of billions of neurons, covers a folded surface area of about one fifth of a square meter, consists of about six cell and fibre layers and has a thickness of a few millimeters. It is subdivided into different functional areas that are in turn structured by graded distributions of molecules as well as by an internal modular organization. Some 100,000 kilometres of neuronal fibres are involved in the connections of the neural network, many of them extending over long distances.

The formation of the network is essentially specified by genes, however indirectly. In brain development, neural tissue expresses celltype-, stage- and position-dependent biochemical markers that are sensed by growing neural fibres - and especially by axonal growth cones at their tips - for directional guidance, branching, and target identification. Some markers are graded and may function analogous to longitudes and latitudes in ocean navigation: "navigating" growth cones sense these signals, transduce them into their internal structures and process them according to the program of the cell of origin of the axon in order to assess the appropriate direction in which to approach the specific target position. This is one of the mechanisms of growth cone navigation that insures that a limited set of genes can organize network formation for a much larger number of fibers to a first approximation. These mechanisms imply that the formation of connections is mediated by the developmental regulation of the synthesis of neuronal proteins. A key role is expected to be played, as explained above, by sets of regulatory sequences in the genome: by interacting with regulatory proteins, the regulatory sequences contribute to organizing the developmental expression of positional markers in neural tissue. Most importantly, sets of regulatory sequences are expected to control the expression of sets of proteins that specify, in a developmentally regulated manner, the different responses of the navigating growth cones to tissue markers for directional growth and appropriate targeting. The set of growth cone proteins involved could be limited, but their developmental regulation must be intricate and complex in accordance with the complexity of the neural networks to be generated. This implies that the organization of the regulatory sections of the genomic DNA which act as microprocessors of spatio-temporal brain development is likely to be a main determinant of the network that is formed, however indirect the relation may be. The fine-tuning of connections is then caused by activity-dependent self-organization.
An indirect but compelling relationship thus exists between, on the one hand, the order of the network of gene regulation (with interconnections mediated by proteinprotein and protein-DNA-interactions in the cell) and, on the other hand, the order of the real neural network (with physical connections by neuronal fibers). We are still far from decoding this relationship, despite much progress in neurogenetics. And yet, there is little doubt that regulatory sections of the genome, as presumably main determinants of how growth cone navigation and targeting are organized, are also main targets for the mutational changes that underlie brain evolution: small changes involving single nucleotides or smaller groups of nucleotides that may, in some cases, suffice to reprogram regulation, but also certain rare, major duplications, transpositions or other rearrangements of genomic DNA.

The basic concept supported in this paper addresses the evolution of novel, or at least highly generalized, capabilities of the human brain. It states that such capabilities could have arisen through novel combinations of existing, possibly complex sets of regulatory sequences, followed by later, more gradual development; an example to which this concept may apply is the evolution of human cognition-based empathy (Gierer 1998). In general, the hypothesis suggests that accidental changes in the modular and synergetic organization of the network of gene regulation may affect the spatio-temporal genetic expression of proteins involved in forming the neural network; this may, in rare cases, enable the rise of novel or significantly upgraded capabilities of information processing in the brain. It seems plausible that the likelihood of such evolution through synergetic combinations of regulatory subroutines increases with the increasing 'sophistication' of the existing sets of such subroutines. This would accord with the apparently rapid evolution of human brain capabilities that led to our current species of Homo sapiens, biologically modern man, some hundred thousand years ago.

\section{Generalizable capabilities of the human brain}

Our species is characterized by culture, that is, by the capability to generate and transfer information of increasing complexity from one generation to the next. Information generates information, such that elaborate cultures can develop and diversify even in the absence of genetic changes. Limited capacities for tradition and learned features are known in animals, and tradition-dependent tool making was a capability of hominids already some million years ago; and yet, the potential for the impressive dynamics of cultural change as it is archeologically well documented from the time of the earliest ivory sculptures (Conrad 2003) and cave paintings about 35,000 years ago up to the invention of agriculture 10,000 years ago and through to modern civilization is hardly much older than 
some hundred thousand years. Cultures can develop independent of genetic change, but the general capability of our species for cultural dynamics must itself be based, however indirectly, on genetic determinants of human brain development and is thus a product of biological evolution. It is remarkable that the genetic analysis of modern man suggests a common origin of the entire human population of today in a small group in Africa some hundred thousand to two hundred thousand years ago. This finding suggests in turn one or a few distinct genetic changes that may have significantly advantaged this line of human evolution over others. Genetic and cultural features probably co-evolved for some time. There are no compelling reasons to assume significant genetic improvements in human brain capabilities over the last few thousand generations.

The most characteristic capabilities of the human brain are related to grammatically structured, symbolic language, and strategic thought. The latter encompasses memories about remote pasts, and imagination about possible distant futures. This includes one's own possible future states and the assessment of their emotional desirability in order to choose proper strategies. Strategic thought requires the brain to contain multiple self-representations, including features of one's own actual and envisaged mental states. Furthermore, humans are capable of cognitionbased empathy - sensing others' mental states including others' emotions - which helps to predict their behaviour and thus upgrades the quality of strategic thought. However, one side effect of vicarious emotions is that they may favour cooperative and altruistic behaviour. Even in the highest animals, empathy is developed to only a rudimentary degree. Human cognition-based empathy appears to be a product of the recent evolution of Homo sapiens. It requires within one's own brain the efficient representation of actual as well as potential mental states of others, and their connection with one's own emotional centers.

\section{The distinct initiation hypothesis}

In explaining the evolution of "higher" brain capabilities, pure gradualism is not excluded on logical grounds. And yet, the increasing evidence about highly sophisticated gene regulation leads us to apply the distinct initiation hypothesis outlined above to, in particular, the evolution of the human brain. Rare novel combinations and integrations of - predominantly regulatory - sections of the genome may have affected patterns of neuronal connections initiating, however indirectly, the evolution of new or much upgraded, widely applicable capabilities of the neural network. Such capabilities may include the levels of abstraction required to apply analytical processes to analytical processing itself; the inclusion of assessments of a person's actual and possible mental states into his or her mental state itself; the introduction, perhaps, of control processes which confine the logical intricacies and ambiguities involved in such self-applications; and the representation of possible mental states of others and their connection to one's own emotions.

It is even conceivable that it was one primary genetic change that initiated the evolution of biologically modern man: the introduction of some novel but subtle feature of connectivity into the cerebral cortex at large which allowed for meta-levels of abstraction and upgraded modes of information processing. This may have set the stage for the evolution of interrelated but diverse higher capabilities of grammatically structured language, symbolic thought, strategic thought, cognition based empathy, and so forth. However, the assumption of such a master switch is not a constitutive part of the more general concepts explained above.

This outlines my arguments in favour of the essential role of few distinct genetic events that initiated the evolution of innovative capabilities of the human brain. This remains a hypothesis, apparently not a mainstream one, but shared, I am sure, by others. It is consistent with the writings of Povinelli and Preuss (1995) on "important differences in how humans, great apes and other animals interpret other organisms", which suggest that "at some point in human evolution, elements of a new psychology were incorporated into existing neural systems". It is even more in line with the statement by Pääbo (2001) in an article on the human genome project that presumably "one or a few genetic accidents made human history possible a realization that will provide us with a whole new set of philosophical challenges to think about". A profound understanding of human evolution will require advances in developmental neural biology in combination with explicit theoretical models. As discussed above, a crucial issue is the indirect relationship between the order of the network of gene regulation involved in neural development and the order of the corresponding neural network that underlies its functional capabilities.

To encourage open-mindedness with respect to this direction of thought I will now, in the second part of this essay, consider biological evolution in terms of a more general concept, namely innovation, including technological innovation.

\section{Biological evolution and technological change: differences and similarities}

It has long been observed by many authors that various aspects of technological innovation lend themselves to analysis in terms of evolutionary concepts ranging from close formal relationships to qualitative analogies and metaphors (Ziman 2000). In search of correspondences bet- 
ween biological evolution and technological innovation it is tempting to consider the history of original technological ideas in evolutionary terms. Indeed, Edison's invention of the telephone, analysed in this way, suggests quasievolutionary steps (Carlson 2000). However, it turns out that the similarities to biological evolution are weak when one focuses on very early, ingenious, but unsuccessful ideas and developments such as the expansion of heated air with applications restricted to stage machinery in the theatres of first-century Alexandria, or the design of flying machines by Leonardo da Vinci in the 15th and 16 th centuries.

In contrast, the economically successful prototypes of new technologies which lead to a new development at large exhibit much closer analogies to biological evolution. They correspond to a genetic change that opens up fitness advantages with the prospect of further development, and the increase of market share corresponds to the increase of the share of a genetic trait in the population. Of particular interest in this context are "long waves" of technological change such as the replacement of wood by coal as an energy source, various technologies of steel processing, and the replacement of sailing vessels by steamboats (Nakicenovic 1986; Marchetti 1988).

Whereas many studies aim at recruiting biological theories of evolution to analyse technological change, my article reverses that direction: can we expect, by studying the history of technology, to approach a better understanding of biological evolution? Technological developments involve gradual improvements as well as distinct initiations. My emphasis will be on combinatorial initiation, and my first example concerns the steamship.

\section{Steamships}

Proposals for combining steam engines and boats date back to the 17th century in Europe. Various boats were actually constructed late in the 18th century; all of them were economic failures. Only after Watt had designed and built an efficient type of steam engine, primarily applied to mining, did economic steamboats seem within reach. At the turn of the century, Symington built the "Charlotte Dundee", a steam-driven tugboat for operation on the Ford and Clyde canal in Scotland. It worked fairly well and was on the verge of economic success when the managers of the canal withdrew their permission on environmental grounds: they feared that the canal banks would be damaged. At the same time, a rich merchant purchased the monopoly for steamship traffic on the Hudson River from the State of New York - for steamships that did not yet exist. Fulton, who had seen Symington's “Charlotte Dundee" in 1801, realized the necessary combination: cooperating with the financer and holder of the steamboat monopoly; buying the most advanced steam engine made by Watt; ordering a very modern version of hull construction; implementing an advanced type of paddle wheel; and bringing all these things together in the "Clermont" in 1807 (figure 2a). Though most observers, including the financer, expected or feared that the contraption would blow up, it worked properly, made money, and initiated the age of the commercial steam ship.

A scant four years later the first steamboat went up the Mississippi, "the" market section with the best opportunities at this early stage. The famous river boats - "big wheel keep on turning" - played a decisive role in developing the land near the river along more than a thousand miles. The increasing demands in turn stimulated ship-building technology, with high-pressure steam engines using fuel more efficiently. High pressure was dangerous: in fifteen years there were 35 explosions with 250 casualties. By 1816 the first steamboat built in Prussia was sailing from the Schlo $\beta$ Bellevue - nowadays residence of the Bundespräsident - to the Potsdam lakes, but this was no economic success. In 1819, the "Savannah" crossed the Atlantic, still mainly dependent on sails, but with the support of a steam engine driving the paddle wheels during part of the trip. This was no economic success either-no paying passenger dared to take part in the voyage - but the feasibility of steam propulsion for ocean crossing was now confirmed. This profoundly altered the "fitness landscape" for further developments and this, in turn, increased the potential of steamboats. Two design innovations were particularly important: the use of iron instead of wood for the hull, and the screw propeller instead of the paddle wheel for propulsion. Both were combined for the first time in 1843 in the "Great Britain" (figure 2b), setting the stage for the spectacular development of ocean-ship building. Gradually, the equipment of steamboats with sails was reduced until sails were eventually abandoned, whereas improved technologies for steam propulsion, and hulls made of steel instead of iron, allowed for increases in size, speed, and luxury up to the days of the famous "Titanic" and beyond.

Remarkable features in this development remind us of features of biological evolution. Initially, the new technique was no substitute for the old one. To the contrary, there was a parallel development of the most advanced constructions of sailing boats. In contrast to the strikingly non-gradual initiation of steamship development by the combination of steam engines and boats, plus the subsequent integration of the screw propeller, sailing ships developed along much more gradualistic pathways, especially if we define "gradualistic" liberally so that it includes increases in numbers of constituents such as sails and masts. The introduction to the exhibits in the excellent "Schiffahrtsmuseum" in Bremerhaven states that "the arrangement of sails was developed, in a simple way easy 

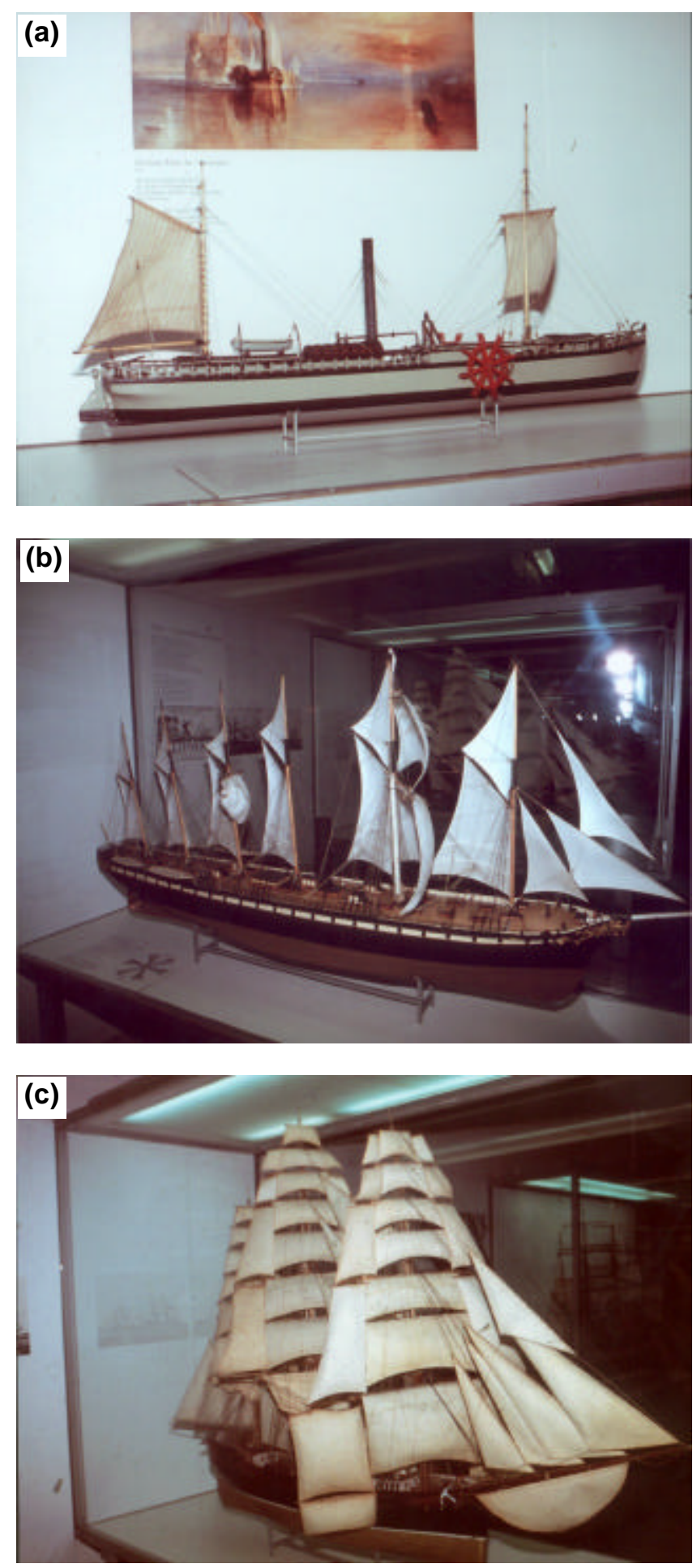

Figure 2. Development of steamships: (a) Fulton's "Clermont" of 1807, the first economically successful combination of steam engine and boat. (b) The "Great Britain", built 1843-1845, combining for the first time a steam engine, iron hull and screw propeller. (c) Clipper "Republic" of 1869. These fast sailing vessels with tall masts and huge sails successfully competed with steamships on the oceans far beyond the middle of the 19th century. (Models: Deutsches Museum München; photographs: W Gierer). to imitate, out of the yard-sail of medieval boats. Large boats, of course, require large sails. First, they were distributed onto more, often onto three masts. Then, the masts were elongated.... and two sails were placed on top of each other. Later, a third and a fourth sail were added . ..". Half a century after the "Clermont"s first trip, it was the slim clippers with tall masts and very large spreads of sails that dominated the scene (figure 2c). During the California gold rush they made the trip from the East Coast to California faster and more safely around Cape Horn than was possible on the overland trip. In the 1860 s spectacular speeds were reached during the annual tea race to China, and only the opening of the Suez Channel signalled the beginning of the end for the age of sailing vessels. And yet, modernized versions of sailing ships, now using steel for their hulls, were built as late as circa 1900 .

Some facets of ocean-ship development remind us of the biological concept of co-evolution. Just two examples: steamships encouraged the development of harbour architecture suited to large boats and allowing for coal supplies en route; such infrastructure, in turn, increased the economic efficiency of larger steamships. Steamship development encouraged the building of the Suez Channel; the Channel, in turn, gave steamships decisive advantages over sailing vessels for connecting Europe and Asia.

Now I would like to come back to more formal aspects of technological development. Figure 3 shows in a logarithmic scale the penetration and saturation of the US market by steamboats. It took more than seventy years for ship propulsion by steam to reach $50 \%$ of market share. Such an extensive time scale is quite typical for major technological innovations - they often take longer than one might expect to penetrate the market. There was a phase of nearly exponential increase after Fulton's "Clermont" of 1807. Many of the associated technological developments accumulated long before that date, but only the synergetic combination of relatively mature components generated the economic success necessary to initiate an exponential "Eigendynamik".

\section{Two more examples: Electricity and containers}

Further impressive examples in the history of technology demonstrate how the specific combination of elements into a system initiates large-scale technological development. Edison's first electric power station of 1882 in New York's Pearl Street combined dynamos driven by steam engines, sophisticated distribution systems, and the design of a functional as well as economic light bulb. This combination initiated the provision of multi-purpose electricity for nearly everybody throughout the world. In a second example, the combination of standardized containers with computerized logistics in the sixties revolutionized the distribution of goods in general, and sea transportation in particular (figure 4). 


\section{Self-regulation, self-organization, self-reference}

A particularly interesting type of specific initiation of a new technology is the invention of feedback mechanisms that allow a system to generate the prerequisites of its own operation. It took about fifty years after Faraday's discovery that electricity can be produced with the aid of magnetism to develop a dynamo that generates its own magnetic field in order to produce electricity. It maintains a residual magnetic field at rest from which to prompt a start-up. This allows powerful fields to build up by selfenhancement. It took time to develop the Jet engine in such a way that it could generate the prerequisite of Jet propulsion - compressed air - in itself. Meeting such requirements of self-regulation and self-organization is often easy to postulate but difficult to achieve. Formally, this reminds us of the intricacies of some self-referential features of human cognition.

\section{A second look at recent human evolution}

In attempting to apply notions and intuitions derived from technology to the evolution of human capabilities such as strategic thought and language, I am emphasizing distinct genetic effects that may lead to synergetic combinations of cues that determine and regulate brain development, thus initiating a new evolutionary direction. I am focusing especially on the period some 300,000 to 100,000 years ago that gave rise to biologically modern man. Admittedly, inferences about the importance of distinct changes during this period and about the key role of regulatory genomic sequences in such changes remain hypothetical. I do not exclude the possibility that gradualism may take us farther in explaining human evolution than I would currently suppose. I think, however, that placing too much faith in gradualism may divert our attention from the most important biological features and their determinants in the human genome.

For example, it is sometimes postulated that in the course of evolution, it was the gradual increase in the size of the human brain that gave rise to its characteristic capabilities, perhaps by allowing for an increasingly greater activity-dependent self-organization of the neural network. Within a century, ship sizes increased by a factor of several times ten, and yet nobody would consider this increase to be an explanation of or for steamships; in-

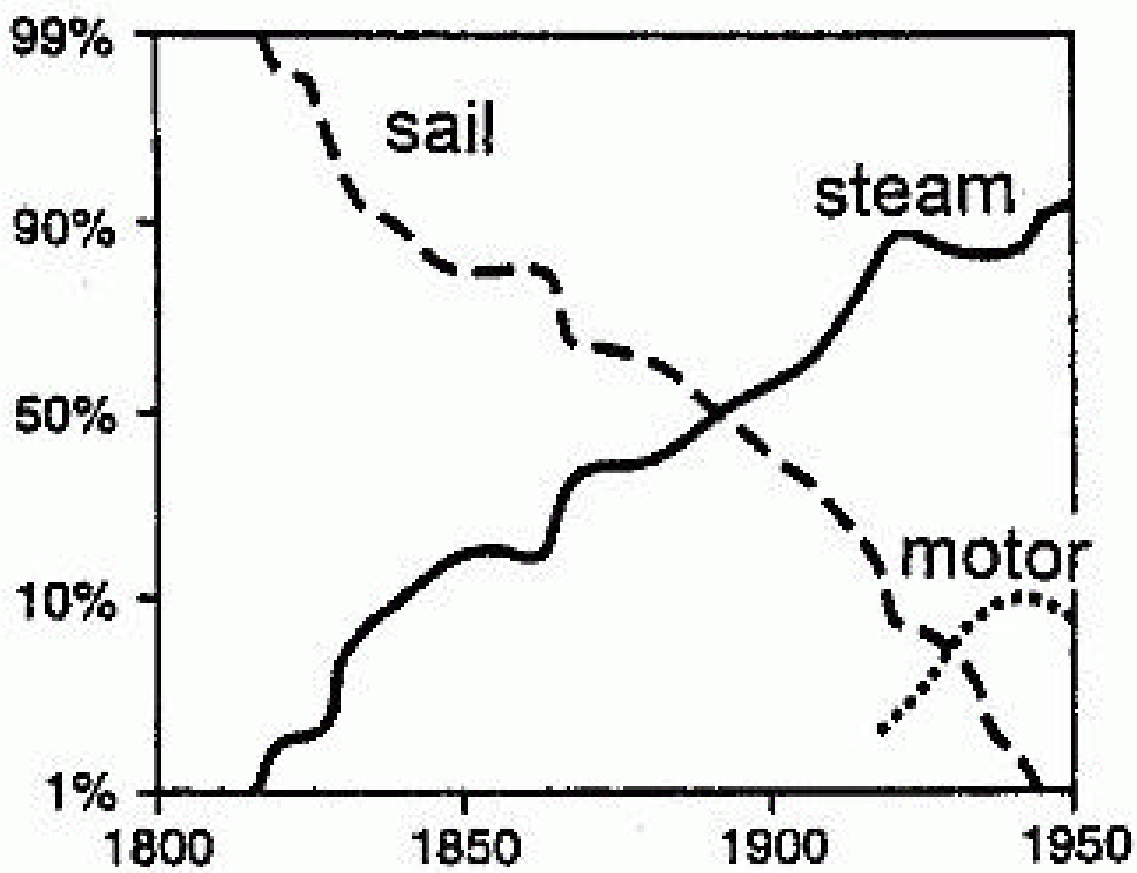

Figure 3. Increase of market share by steam ships (in terms of the US merchant tonnage), plotted in a logarithmic scale, according to Nakicenovic (1986) and Marchetti (1988). The initiation of commercial steamboat traffic was followed by a continuous increase. However, it took some 70 to 80 years until the market share of steamships exceeded the 50\% mark, overtaking the market share of sailing vessels. Such long waves are typical for major technological changes and exhibit formal analogies to the dynamics of the spread of favourable mutations in a population. 
stead, it was the new technology of steam propulsion that rendered large ships technically and economically feasible. Likewise, in brain evolution, it is reasonable to assume that certain upgraded capabilities of the neural network were the primary cause for increased fitness via subsequent increases in brain size.

One important aspect of brain development, especially in higher organisms, is the involvement of activity-dependent processes in the formation and functioning of neural networks. Some of them display features of internal selforganization, others are driven by external - for instance visual - stimuli. However, this does not imply that genetics do not matter in understanding higher brain functions. In the course of development, the early though sometimes crude formation of the neural networks often precedes electrical activity, and is dominated by the processes of tissue patterning and axonal guidance described above. The basic order of the nervous system in the brain and its connectivity are laid down in this manner. These mechanisms contribute to speed and reliability in acquiring functional performance. They are then complemented by processes depending on electrophysiological activity that are effective for generating precise neural connections and the acuity of functions, and that allow for adaptations to changing conditions. The complementary benefits of these mechanisms support the notion that evolutionary forces themselves tend to balance their relative contributions for optimizing the biological fitness of the organism as a whole in a species-specific manner (Gierer and Mueller 1995). It is emphasized that the activity-dependent processes are by no means independent of genetic instructions. In fact, self-organizational capabilities and their outcome under normal conditions are indirectly but nevertheless crucially influenced by genetically encoded boundary conditions that determine whether, where, when and how these processes occur in the course of development.

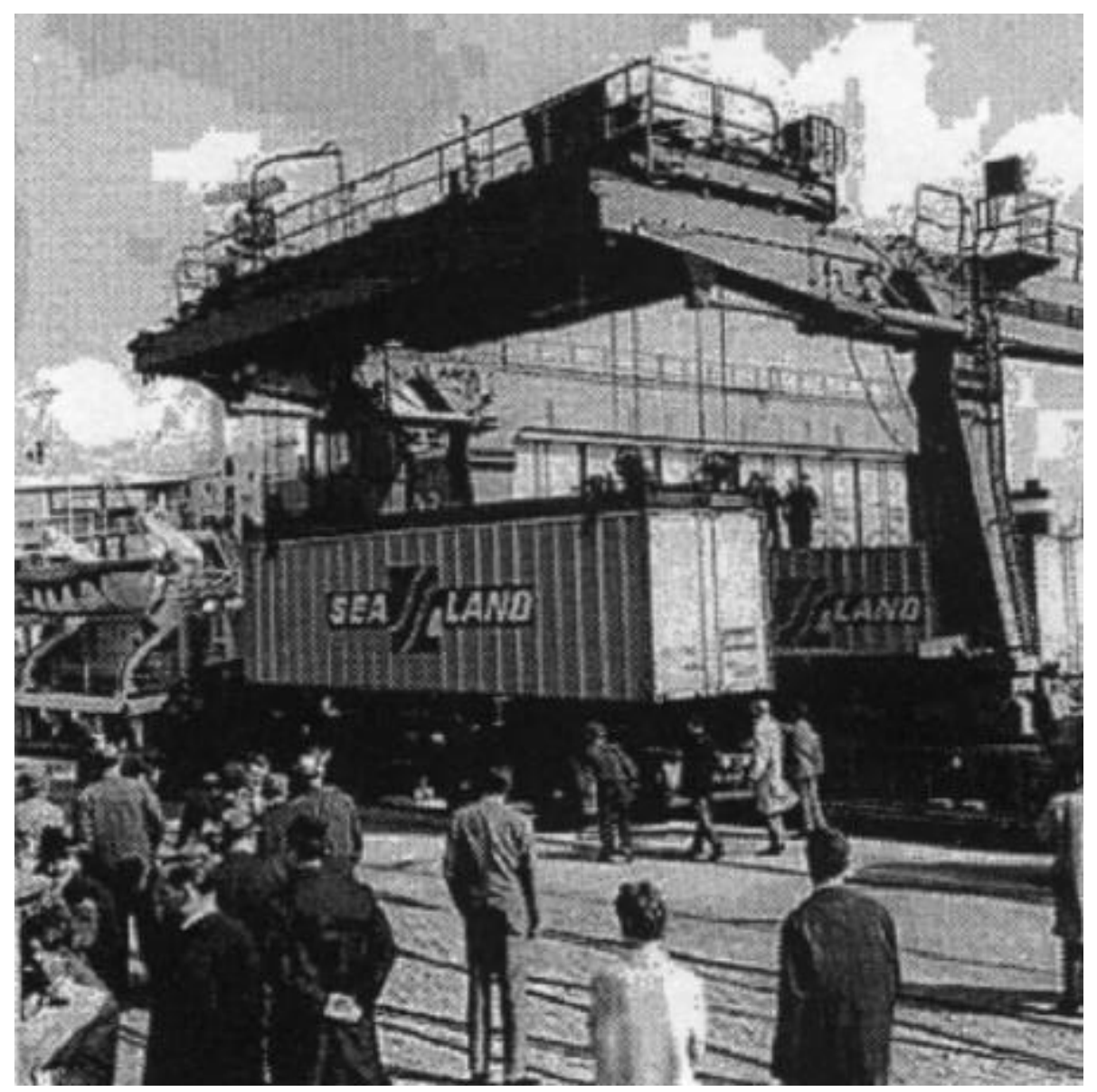

Figure 4. Container logistics. In the last four decades, the introduction of standardized containers guided by computerized logistics has revolutionized the distribution of goods in general, and of sea transportation in particular. This image records the arrival of the first container of the Sea-Land company in the harbour of Bremen, in 1966. 


\section{In conclusion: a plea for open-mindedness}

In summary, my argument suggests that chance mutations in human evolution occasionally resulted in highly specific combinations of the subroutines of gene regulation that are involved in brain development, and that some of these led to synergetic if not qualitatively novel capabilities of the neural network. While their initial phenotypic effects were presumably small, they may have opened up a new direction of evolution to be followed by many further genetic changes. Thus, Darwin's original notion implying that phenotypic modifications of genetic variants are small is presumably correct. The emphasis on nothing but the accumulation of frequent common steps for explaining evolution would not, on the other hand, do justice to the presumably crucial role novel combinations of regulatory genomic sequences may play in specific initiations of new directions. This combinatorial hypothesis is supported by analogies with important episodes in the history of technology, and is suggested here to hold, in particular, for the evolution of some of the sophisticated capabilities of the human brain. Though one may argue about the use of hidden analogies between biological evolution and history of technology, a look at the latter may at least help somewhat to open our minds, and to broaden our perspectives in dealing with such types of questions.

\section{References}

Alberts B, Johnson A, Lewis J, Raff M, Roberts K and Walter P 2002 Molecular biology of the cell 4th edition, (New York: Garland Publishing Inc.)

Arnone M J and Davidson E H 1997 The hardwiring of development: organization and function of genomic regulating systems; Development 124 1851-1864

Carlson W B 2000 Invention and evolution: the case of Edison's sketches of the telephone; in Technological innovation as an evolutionary process (ed.) J Ziman (Cambridge: Cambridge University Press) pp 137-158
Conrad N J 2003 Paleolithic ivory sculptures from southwestern Germany and the origins of figurative art; Nature (London) 426 830-832

Davidson E H et al 2002 A genomic regulatory network for Development; Science 295 1669-1678

Darwin C 1859 On the origin of species by means of natural selection (London: Murray) pp 71, 189

Gierer A 1973 Molecular models and combinatorial principles in cell differentiation and morphogenesis; Cold Spring Harb. Symp. Quant. Biol. 38 951-961

Gierer A 1998 Networks of gene regulation, neural development and the evolution of general capabilities such as human empathy; Z. Naturforsch. C53 (Special issue: Natural organisms, artificial organisms, and their brains) 716-722

Gierer A and Mueller C M 1995 Development of layers, maps and modules; Curr. Topics Neurobiol. 5 91-97

Haring N 1955 The creation of the world according to Thierry; Archives d'Histoire Doctrinal et Litteraire de Moyen Age 146169

Keys D N, Lewis D L, Selegue J E, Pearson B J, Goodrich L V, Johnson R L, Gates J, Scott M P and Carrol S B 1999 Recruitment of a hedgehog regulatory circuit in Butterfly eyespot evolution; Science 283 532-534

Marchetti C 1988 The future; in Synergetics and dynamic instabilities (eds) C Caglioti and H Haken (Amsterdam: North Holland) pp 400-416

Mayr E 1988 Toward a new philosophy of biology (Cambridge: The Belknap Press of Harvard University Press) pp 170; 410-411

Nakicenovic N 1986 Patterns of change: Technological substitution and long waves in the United States (Working paper 86-13, International Institute for Applied Systems Analysis, Laxenburg, Austria)

Pääbo S 2001 The human genome and our view of ourselves; Science 291 1219-1220

Pires daSilva A and Sommer R J 2003 The evolution of signalling pathways in animal development; Nature Rev. Genet. 4 39-49

Povinelli D J and Preuss T M 1995 Theory of mind: Evolutionary history of a cognitive specialization; Trends Neurosci. 18 418-424

Tautz D 2000 Evolution of transcriptional regulation; Curr. Opin. Genet. Dev. 10 575-579

Wilkins A S 2002 The evolution of developmental pathways (Sunderland: Sinauer Associates)

Ziman J M 2000 Technological innovation as an evolutionary process (Cambridge: Cambridge University Press) 